\title{
Fields and Trajectories in the Magnicon
}

\author{
Paul Tallerico and Daniel Rees \\ Los Alamos National Laboratory \\ Los Alamos, NM 87545
}

\section{Abstract}

The magnicon is a new type of deflection-modulated microwave amplifier that has great potential for high-power, high-efficiency microwave generation. The magnicon has two $\mathrm{TM}_{110}$ cavities, each with fields rotating at microwave frequencies. The first cavity deflection-modulates an clectron beam into an expanding spiral path, and the second extracts kinetic energy from the modulated beam. Static magnetic fields employed in the second cavity produce cyclotron motion at frequencies that are multiples of the microwave frequency. The results of calculations performed on this beam-wave system, with a first-order numerical model, provide guidance for magnicon experimental designs. Field calculations have also been done with the three-dimensional electromagnetic field simulation code, MAFIA. The field and trajectory calculations demonstrate that very high output power and dcto-rf conversion efficiencies are possible with the magnicon.

\section{INTRODUCTION}

The magnicon is a deflection-modulated RF amplifier that promises to reach new levels of efficiency and power. The magnicon is an improved version of the gyrocon [1], in which the interaction in the output cavity involves cyclotron resonance. The cyclotron resonant interaction may be distributed, rather than concentrated, so that much lower output cavity fields are required in the magnicon than in the gyrocon. A limitation of the magnicon (and of all other gyroresonant devices) is that only the transverse energy of the electron beam is converted into microwaves. However, just as the gyroresonant interaction in linear beam tubes (the gyrotron) has increased the power and frequency limits in linear beam technology, the distributed interaction in the magnicon will increase these limits for deflection-modulated devices.

\section{THEORY OF OPERATION}

A schematic drawing of a magnicon is shown in Figure 1. Many magnicon architectures are possible, such as several deffection cavities or static magnetic fields in the deflection rogion. A solid electron beam is produced and accelerated to a lairly high voltage (above $100 \mathrm{kV}$ ), and the beam passes through a cylindrical TM110-mode deflection cavity. This cavity supports, on its axis, a rotating magnetic field that is perpendicular to the beam axis. The deflection-cavity fields cause the beam trajectories to follow an expanding spiral. A sketch of these fields is shown in Figure 2. After a drift

\footnotetext{
*Work supported and funded by the US Department of Defense, Army Strategic Defense Command, under the auspices of the US Department of Energy.
}

distance that makes the spiral large enough, the beam is injected into a $\mathrm{TM}_{110}$-mode cylindrical output cavity. The output cavity contains an axial magnetic field such that the beam's cyclotron frequency is equal to the output cavity resonant frequency. The if fields in the output cavity must also rotate at the cyclotron frequency (or a harmonic). The ideal magnetic field distribution is a step function in $\mathrm{z}$, because with this field a maximum amount of the beam's axial kinetic energy is converted into rotational energy. In the absence of $\mathrm{rf}$ fields, the spiral trajectories in the output would all cross the axis, when if fields are present, the electrons give up energy to the rf fields during the decelerating phase of the cyclotron period, but during the accelerating phase, the electrons are near the axis, where the axial $\mathrm{E}$ field is small, and are accelerated by only a small amount.

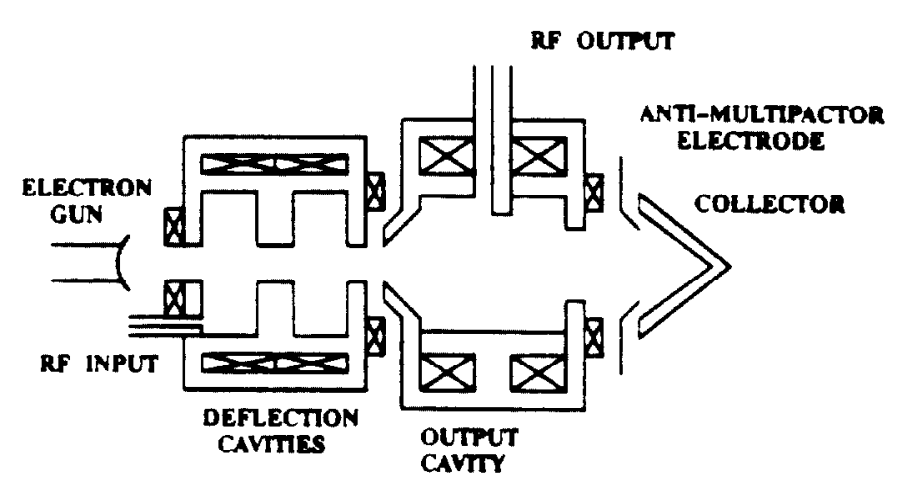

Figure 1. Magnicon Schematic Diagram

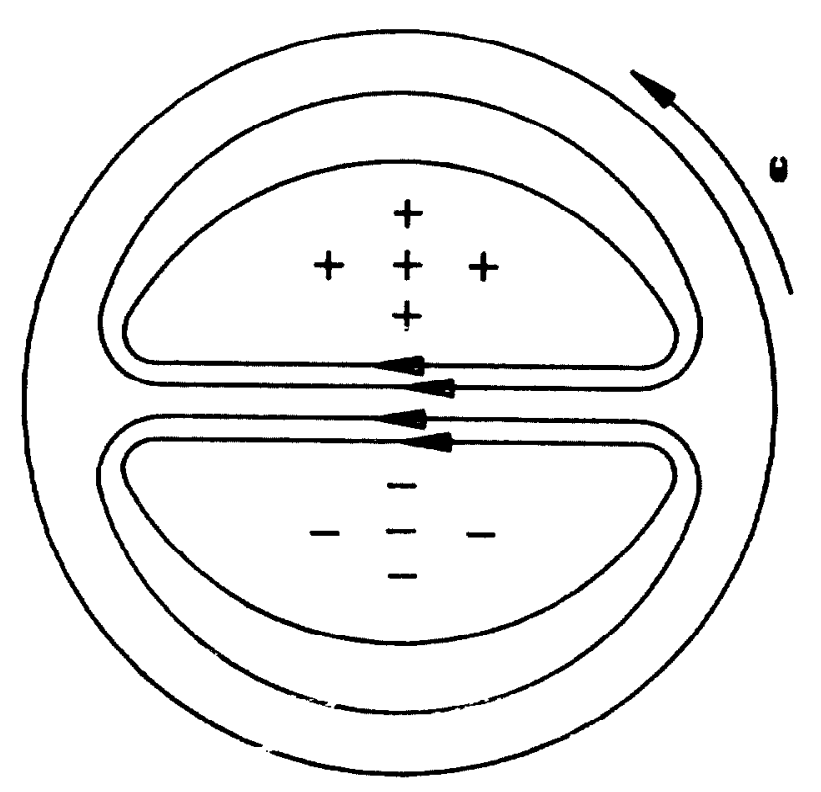

Figure 2. Cross Sectional Plot of Deflection Cavity Fields 
Since the cyclotron and output cavity frequencies are cqual, the energy exchange process may continue over several rf cycles. Thus, the values of fields in the output cavity are small, and the cavity walls are not heated very much. This allows either very high frequency operation with the magnicon (10 to $20 \mathrm{GHz}$ appear possible), or very high CW output (powers of over $1 \mathrm{MW}$ are possible over the .5 to $2 \mathrm{GHz}$ frequency band). There are now working magnicons that have produced $2.8 \mathrm{MW}$ at an efficiency of $73 \%$ in pulsed systems. In view of their high efficiency, magnicons seem very well suited both to the next-generation linear collider applications (at $10-100 \mathrm{MW}$ in 100-ns pulses at frequencies near 20 $\mathrm{GHz}$ ), and for proton accelerators with $1 \mathrm{MW} \mathrm{cw}$ at $0.5-2$ $\mathrm{GH} z$. Another advantage of the magnicon is that the output beam is much more monoenergetic than other linear beam devices. This is a result of the ratio of electric to magnetic rf ficlds in the output resonator. Although the energy is only extracted by the axial electric field, the transverse magnetic ficlds reduce the size of the cyclotron orbit as the beam is decelerated, so that the transverse energy appears as longitudinal energy. 'Thus, the axial energy remains constant, and the magnicon is a very good candidate for collector depression.

\section{MAgNiCON MODEL}

We developed a relativistic model of the magnicon that calculates single-particle trajectories for the deflection cavity, drift space, half cusp, and output cavity. The model assumes a zcro-emittance beam and neglects space charge effects. For computational simplicity, the model uses square deflection and oulpul cavities of width a, resonant at a frequency $w$, in the $\mathrm{TM}_{120}$ mode. The fields for this mode are given by [2]

$$
\begin{aligned}
& E_{z}=w B_{120} \sin (\pi x / a) \sin (2 \pi y / a) \\
& B_{x}=(2 \pi / a) B_{120} \sin (\pi x / a) \cos (2 \pi y / a) \\
& B_{y}=-(\pi / a) B_{120} \cos (\pi x / a) \sin (2 \pi y / a),
\end{aligned}
$$

where $B_{120}$ is related to the peak rf magnetic field amplitude for the TM120 mode. A rotating rf field is modeled in the deflection and output cavities by the superposition of two $\mathrm{TM}_{120}$ modes excited 90 degrees out of phase on adjacent cavity walls. In the model, the particle is deflected off-axis in the deflection cavity and enters a drift space, where it drifts approximatcly one Larmor diameter off-axis. The particle then enters a half-cusp solenoidal field approximated by a $B_{Z}$ componenl given by

$$
\mathrm{B}_{\ell}=\mathrm{B}_{0}\left(1-\mathrm{e}^{-2 / 20}\right),
$$

where zo controls the rate of rise of the $\mathrm{z}$-directed component of the static field, and $B_{O}$ is selected such that the cyclotron frequency of the single particle is nearly equal to the rf frequency, $w_{c}$, given by [3]

$$
B_{0}=\partial m_{O} w_{c} /|q|,
$$

where $m_{O}$ is the particle rest mass, $\partial$ represents gamma, and $q$ is the particle charge. The radial components of the static field are calculated using the paraxial approximation [4]. As the particle enters the half-cusp field, a transfer of energy takes place from the longitudinal to the transverse direction, and the transverse energy is then extracted by a long-term interaction with the rotating if fields.

The results of the single-particle simulation are included in Fig. 3 through 6. Figure 3 is a $x-z$ plot of the particles position from deflection cavity to output. Figure 4 is an $x-y$ scatter plot of the single-particle trajectory. Figure 5 is a plot of the $\mathrm{z}$ position of the particle as a function of time. Figure 6 is a plot of the particle's energy as a function of distance. From Figures 3 and 4 the particle's path as it leaves the deflection cavity and enters the drift space can be observed.

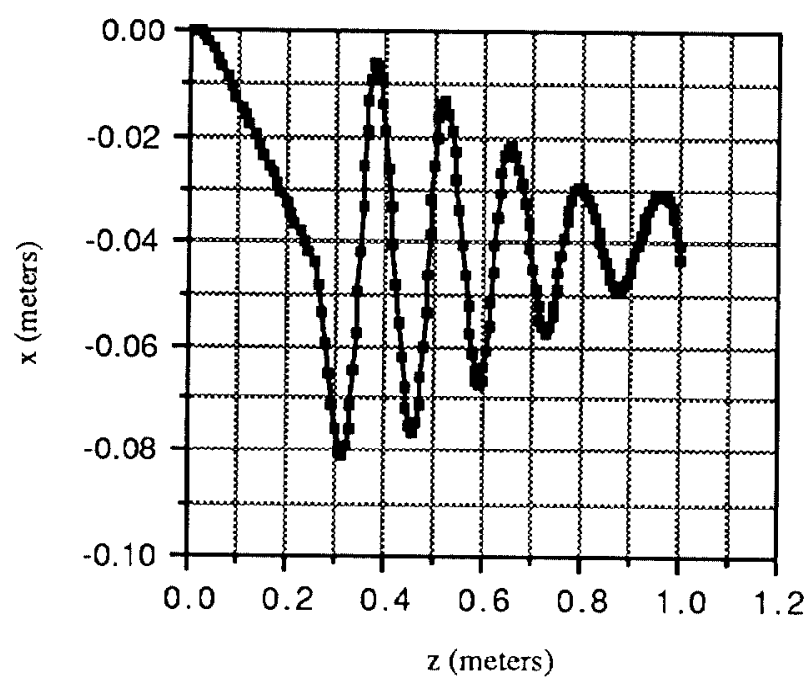

Figure 3. Particle Position from Deflection Cavity to Output

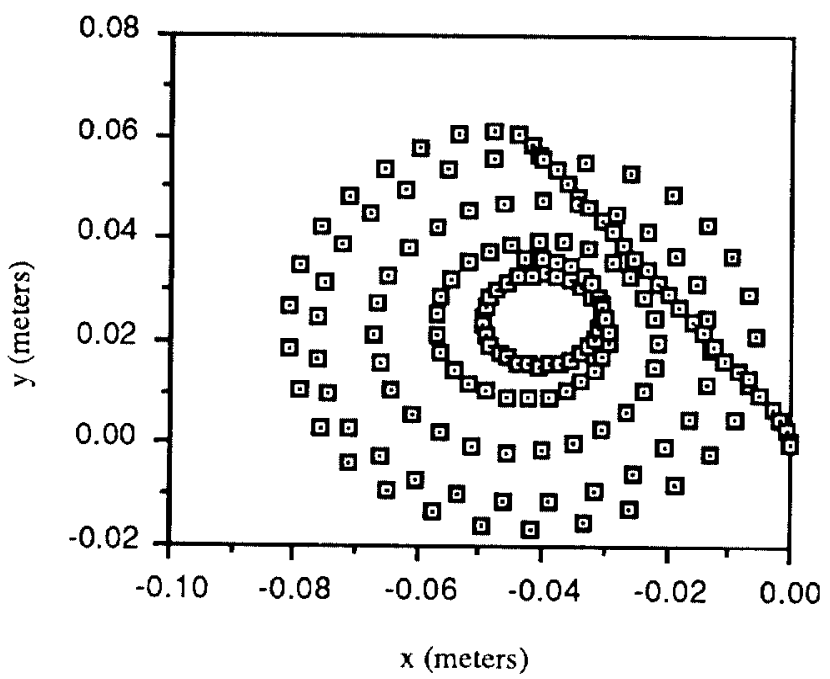

Figure 4. x-y Scatter Plot of Particle Trajectory

As the particle enters the half cusp, it is focused back towards the axis of the device. The decreasing spiral in the figure shows that the particle is giving up its transverse energy. From Figure 5, it is observed that the slope of the particle trajectory in the output cavity, and thus the longitudinal velocity of the particle is constant . 


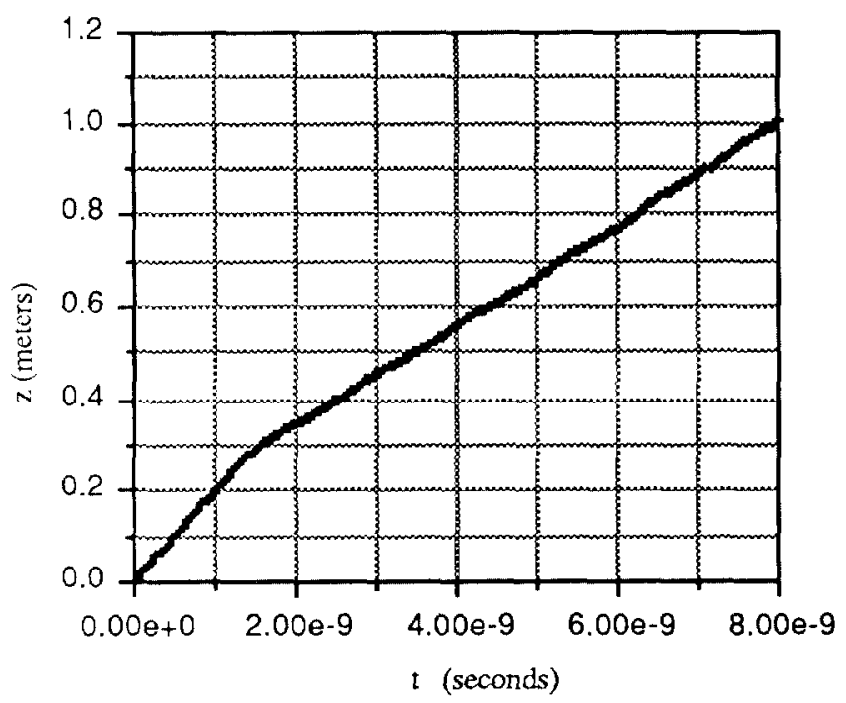

Figure 5. Particle Position as a Function of Time

The constant longitudinal velocity results from the addition of velocity (by the transverse magnetic field when the particle is near the axis) and the subtraction of an equal velocity (by the rotating $E$ field when the particle is farthest from the axis). This addition / subtraction of velocity takes place on cach spiral cycle. As was stated earlier, since the beam is shown to be nearly monoenergetic, the overall cfficiency of the device may be improved by depressing the collector.

Figure 6 illustrates the energy of the particle relative to the input energy.

It can be seen from the figure that beam loading takes place in the deflection cavity, which will increase drive-power requirements; and that energy is extracted during a long-term interaction in the output cavity. The periodic nature of the energy plot results from the electrons' alternately being cxposed to accelerating and decelerating electric fields. The phase of the rotating if field is such that the electrons see the decclerating elcetric field when they are off axis where the electric field is large and are near the axis when the rf fields have rotated by 180 degrees presenting the electrons with an accelerating field. However, as more energy is extracted from the transverse energy of the electrons, they become farther off-

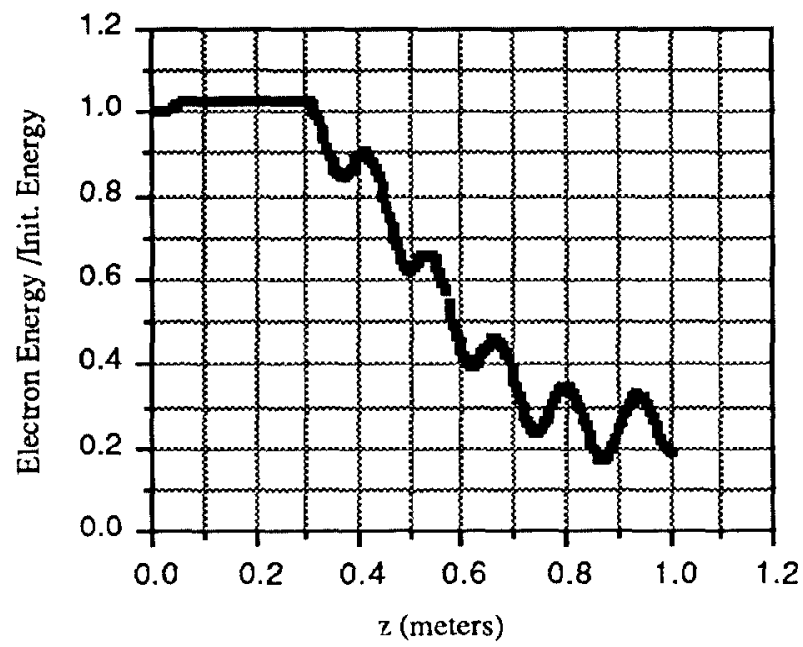

Figure 6. Particle Energy Relative to Initial Energy as a Function of Particle Position

axis during the presence of the accelerating fields. Thus, the periodic variations become more pronounced as more transverse energy is removed from the electrons. Figure 6 shows that electron efficiency averages $75 \%$.

\section{REFERENCES}

[1] G.I. Budker et al, "The Gyrocon - An Efficient Relativistic High-Power UHF Generator," Particle Accelerators $10,41-59,1979$.

[2] N.M. Karliner et al, "The Magnicon - An Advanced Version of the Gyrocon," Nuclear Instruments and Methods in Physics Research A269, 459-473, 1988.

[3] Constantine A. Balanis, Advanced Engineering Electromagnetics, (Wiley New York, 1989), pp. 388394.

[4] Stanley Humphries, Principles of Charged Particle Acceleration, (Wiley New York, 1986), pp. 108-115. 